\title{
TOWARDS THE KOHAYAKAWA-KREUTER CONJECTURE ON ASYMMETRIC RAMSEY PROPERTIES
}

\author{
FRANK MOUSSET, RAJKO NENADOV, AND WOJCIECH SAMOTIJ
}

\begin{abstract}
For fixed graphs $F_{1}, \ldots, F_{r}$, we prove an upper bound on the threshold function for the property that $G(n, p) \rightarrow\left(F_{1}, \ldots, F_{r}\right)$. This establishes the 1-statement of a conjecture of Kohayakawa and Kreuter.
\end{abstract}

\section{INTRODUCTION}

Given a graph $G$, a positive integer $r$, and graphs $F_{1}, \ldots, F_{r}$, we write

$$
G \rightarrow\left(F_{1}, \ldots, F_{r}\right)
$$

if for every colouring of the edges of $G$ using colours from the set $[r]:=\{1, \ldots, r\}$, there exists a copy of $F_{i}$ in $G$ whose all edges have been coloured $i$, for some $i \in[r]$. We study the asymptotic probability that

$$
G(n, p) \rightarrow\left(F_{1}, \ldots, F_{r}\right)
$$

for fixed graphs $F_{1}, \ldots, F_{r}$, where $G(n, p)$ is the binomial random graph with $n$ vertices and edge probability $p$.

An important special case of this problem, known as the symmetric case, arises when the graphs $F_{1}, \ldots, F_{r}$ are all the same. The study of symmetric Ramsey properties in random graphs was initiated by Łuczak, Ruciński, and Voigt [15], who proved that $p=n^{-1 / 2}$ is a threshold for the property $G(n, p) \rightarrow\left(K_{3}, K_{3}\right)$. (The earlier work of Frankl and Rödl [4] established that $G(n, p) \rightarrow\left(K_{3}, K_{3}\right)$ under the stronger assumption that $p \geqslant n^{\varepsilon-1 / 2}$.) This was followed by a series of papers by Rödl and Ruciński [19, 20, 21] that culminated in the following statement. For a nonempty graph $F$, let $d_{2}(F):=1 / 2$ if $F=K_{2}$ and $d_{2}(F):=\frac{e_{F}-1}{v_{F}-2}$ otherwise and define the 2-density of $F$ by

$$
m_{2}(F):=\max \left\{d_{2}\left(F^{\prime}\right): F^{\prime} \subseteq F \text { with } e_{F^{\prime}} \geqslant 1\right\} .
$$

Theorem 1 ([21]). Let $r \geqslant 2$ and suppose that $F$ is a nonempty graph such that at least one component of $F$ is not a star or (in the case $r=2$ ) a path of length three. Then there exist positive constants $c$ and $C$ such that

$$
\lim _{n \rightarrow \infty} \mathbb{P}(G(n, p) \rightarrow(\underbrace{F, \ldots, F}_{r \text { times }}))= \begin{cases}0 & \text { if } p \leqslant c n^{-1 / m_{2}(F)} \\ 1 & \text { if } p \geqslant C n^{-1 / m_{2}(F)} .\end{cases}
$$

One usually refers to the assertion of Theorem 1 for $p \leqslant c n^{-1 / m_{2}(F)}$ as the 0 -statement and to the assertion for $p \geqslant C n^{-1 / m_{2}(F)}$ as the 1-statement. It is worth pointing out that the assumption on the structure of $F$ is necessary. Indeed, if every component of $F$ is a star, then it is easy to see that $G \rightarrow(F, \ldots, F)$ as soon as $G$ has sufficiently many vertices of degree $r\left(\Delta_{F}-1\right)+1$. The function $n^{-1-1 /\left(r\left(\Delta_{F}-1\right)+1\right)}$ is a threshold for this property in $G(n, p)$; on the other hand, $m_{2}(F)=1$ for every such $F$. In the case where $r=2$ and at least one component of $F$ is a path of length three while the others are stars, the 0 -statement of Theorem 1 is no longer true. For example, if $p=c n^{-1 / m_{2}\left(P_{3}\right)}=c n^{-1}$ for some $c>0$, then the probability that $G(n, p)$ contains a cycle of length five with an edge pending

Date: 7th May 2020.

Research supported in part by the Israel Science Foundation (ISF) grants 1147/14 (FM and WS) and 1028/16 (FM) and ERC Starting Grant 633509 (FM).

Part of the work was done while the second author was visiting Tel Aviv University.

A first draft of this paper was produced at the workshop of the research group of Angelika Steger in Buchboden in July 2018. 
at every vertex is bounded from below by a positive constant (that depends on $c$ ); it is easy to check that every colouring of the edges of this graph with two colours yields a monochromatic path of length three. This exceptional case, originally missed by Rödl and Ruciński in [21], was eventually noticed and corrected by Friedgut and Krivelevich [5]; the corrected version of the 0-statement requires the assumption that $p=o\left(n^{-1 / m_{2}(F)}\right)$. A short proof of Theorem 1] was given by Nenadov and Steger [18].

In the case where $F$ is a tree (other than a star or the path of length three) [5], a triangle [6] or, more generally, a strictly 2-balanced 1 graph that can be made bipartite by removing some edge [24, it is known that the property $G(n, p) \rightarrow(F, F)$ has a sharp threshold:

Theorem 2 ([5, 6, 24]). Suppose that $F$ is either (i) a tree that is not a star or the path of length three or (ii) a strictly 2-balanced graph with $e_{F} \geqslant 2$ edges that can be made bipartite by removing some edge. Then there exist $c_{0}, c_{1}$, and a function $c: \mathbb{N} \rightarrow\left[c_{0}, c_{1}\right]$ such that

$$
\lim _{n \rightarrow \infty} \mathbb{P}(G(n, p) \rightarrow(F, F))= \begin{cases}0 & \text { if } p \geqslant(1+\varepsilon) c(n) n^{2-1 / m_{2}(F)} \\ 1 & \text { if } p \leqslant(1-\varepsilon) c(n) n^{2-1 / m_{2}(F)}\end{cases}
$$

for every positive constant $\varepsilon$.

It is widely believed that one can choose $c(n)$ to be a constant function; however proving this and, what is more, determining the value of the constant, remains a formidable challenge.

The main topic of this paper is the asymmetric case of the Ramsey problem in $G(n, p)$, where the graphs $F_{1}, \ldots, F_{r}$ are allowed to be different. This problem was first considered by Kohayakawa and Kreuter [12]. For nonempty graphs $F_{1}$ and $F_{2}$ with $m_{2}\left(F_{1}\right) \geqslant m_{2}\left(F_{2}\right)$, we define the asymmetric 2 -density

$$
m_{2}\left(F_{1}, F_{2}\right):=\max \left\{\frac{e_{F_{1}^{\prime}}}{v_{F_{1}^{\prime}}-2+1 / m_{2}\left(F_{2}\right)}: F_{1}^{\prime} \subseteq F_{1} \text { with } e_{F_{1}^{\prime}} \geqslant 1\right\} .
$$

The following generalisation of Theorem 1 is a slight rephrasing of a conjecture made by Kohayakawa and Kreuter [12]. (The original conjecture was stated only for two colours and it lacked the assumption that the graphs $F_{1}$ and $F_{2}$ are not forests, which was later added by Kohayakawa, Schacht, and Spöhel [14.)

Conjecture 3. Let $r \geqslant 2$ and suppose that $F_{1}, \ldots, F_{r}$ are graphs with $m_{2}\left(F_{1}\right) \geqslant \cdots \geqslant m_{2}\left(F_{r}\right)$ and $m_{2}\left(F_{2}\right)>1$. Then there are positive constants $c$ and $C$ such that

$$
\lim _{n \rightarrow \infty} \mathbb{P}\left(G(n, p) \rightarrow\left(F_{1}, \ldots, F_{r}\right)\right)= \begin{cases}0 & \text { if } p \leqslant c n^{-1 / m_{2}\left(F_{1}, F_{2}\right)} \\ 1 & \text { if } p \geqslant C n^{-1 / m_{2}\left(F_{1}, F_{2}\right)} .\end{cases}
$$

The assumption that $m_{2}\left(F_{2}\right) \geqslant 1$ is necessary, since otherwise we have $m_{2}\left(F_{2}\right)=1 / 2$ (i.e., $F_{2}$ is a matching) and so $m_{2}\left(F_{1}, F_{2}\right)=e_{F_{1}^{\prime}} / v_{F_{1}^{\prime}}$ for some nonempty subgraph $F_{1}^{\prime} \subseteq F_{1}$. In this case, for every constant $C>0$, the probability that $G(n, p)$ with $p=C n^{-1 / m_{2}\left(F_{1}, F_{2}\right)}$ contains no copies of $F_{1}$ at all exceeds a positive constant (that depends on $C$ ); see, for example [11]. The assumption that $m_{2}\left(F_{2}\right)>1$ (which holds if and only if $F_{2}$ is not a forest) is most likely not always necessary, but it precludes exceptional sequences $F_{1}, \ldots, F_{r}$ such as a sequence of stars.

There have been several attempts at resolving Conjecture 3. Kohayakawa and Kreuter [12] proved it in the case where each $F_{i}$ is a cycle. Marciniszyn, Skokan, Spöhel, and Steger [16] observed that the proof of the 1-statement of Conjecture 3 given in [12] for sequences of cycles extends to all sequences $F_{1}, \ldots, F_{r}$ such that $F_{1}$ is strictly 2-balanced, assuming the so-called KŁR (Kohayakawa- LuczakRödl) conjecture [13] holds. (The KŁR conjecture has since been verified, see [1, 3, 23].) The main result of [16] however was a proof of the 0 -statement of Conjecture 3 in the case where each $F_{i}$ is a complete graph. A self-contained (i.e., not relying on the KŁR conjecture) proof of the 1-statement of Conjecture 3 for $r=2$ that assumes a similar density condition, namely that $F_{1}$ is strictly balanced w.r.t. $m_{2}\left(\cdot, F_{2}\right)$, was given by Kohayakawa, Schacht, and Spöhel [14]. This result was generalised

\footnotetext{
${ }^{1}$ A nonempty graph $F$ is said to be 2-balanced if $d_{2}(F)=m_{2}(F)$ and strictly 2-balanced if in addition $d_{2}\left(F^{\prime}\right)<m_{2}(F)$ for every nonempty proper subgraph $F^{\prime} \subseteq F$.
} 
by allowing $F_{1}, \ldots, F_{r}$ to be uniform hypergraphs and extended from two to an arbitrary number of colours by Gugelmann, Nenadov, Person, Škorić, Steger, and Thomas [7]. It was furthermore shown in [7] that the 1-statement of Conjecture 3 holds, with no additional conditions on the graphs $F_{1}, \ldots, F_{r}$, under the stronger assumption that $p \geqslant C n^{-1 / m_{2}\left(F_{1}, F_{2}\right)} \log n$. The proofs of both these statements employed the hypergraph container method developed by Balogh, Morris, and Samotij [1] (see also [2]) and, independently, by Saxton and Thomason [23]. Let us also mention that the short argument of Nenadov and Steger [18] that establishes the 1-statement of Theorem 1 can be rewritten almost verbatim to give a proof of the 1 -statement of Conjecture 3 in the case where $m_{2}\left(F_{1}\right)=m_{2}\left(F_{2}\right)$, with no further conditions on $F_{1}$ (this was explicitly observed in [8]).

In summary, all previous results related to the 1-statement of Conjecture 3 require either some nontrivial assumptions on $F_{1}$ (or both $F_{1}$ and $F_{2}$ ) or a stronger lower bound on $p$. Our main contribution is a proof of this statement in its full generality.

Theorem 4. Let $r \geqslant 2$ and suppose that $F_{1}, \ldots, F_{r}$ are graphs with $m_{2}\left(F_{1}\right) \geqslant \cdots \geqslant m_{2}\left(F_{r}\right)$ and $m_{2}\left(F_{2}\right) \geqslant 1$. Then there exists a positive constant $K$ such that if $p=p(n) \geqslant K n^{-1 / m_{2}\left(F_{1}, F_{2}\right)}$, then

$$
\lim _{n \rightarrow \infty} \mathbb{P}\left(G(n, p) \rightarrow\left(F_{1}, \ldots, F_{r}\right)\right)=1 .
$$

\section{PRELIMinaries}

2.1. Ramsey's theorem. The following quantitative version of Ramsey's theorem can be obtained from the usual version by a standard averaging argument (see, e.g., [7, Theorem 11]).

Lemma 5 (Ramsey's theorem). For every positive integer $r$ and all graphs $F_{1}, \ldots, F_{r}$, there exists a positive $\alpha$ and some $n_{0}$ such that the following holds for all $n \geqslant n_{0}$. For every colouring of the edges of $K_{n}$ with colours from $[r]$, there exists a colour $i \in[r]$ such that $K_{n}$ contains at least $\alpha n^{v_{F_{i}}}$ copies of $F_{i}$ whose edges all have colour $i$.

2.2. Hypergraph containers. The following lemma is a well-known consequence of the hypergraph container theorems obtained by Balogh, Morris, and Samotij [1] and, independently, by Saxton and Thomason [23].

Lemma 6. For every graph $F$ and every positive $\varepsilon$, there exists a positive $C=C(F, \varepsilon)$ such that the following holds for all $n \in \mathbb{N}$. Let $\mathbf{F}(n)$ be the family of all $F$-free graphs with vertex set $[n]$. Then there exist functions

$$
g: \mathbf{F}(n) \rightarrow 2^{E\left(K_{n}\right)} \quad \text { and } \quad f: 2^{E\left(K_{n}\right)} \rightarrow 2^{E\left(K_{n}\right)}
$$

such that, for every $G \in \mathbf{F}(n)$,

(i) $g(G)$ has at most $C n^{2-1 / m_{2}(F)}$ edges,

(ii) $f(g(G))$ contains at most $\varepsilon n^{v_{F}}$ copies of $F$, and

(iii) $g(G) \subseteq G \subseteq f(g(G))$.

\section{Proof overview}

Suppose that $G \nrightarrow\left(F_{1}, \ldots, F_{r}\right)$, that is, that there exists a colouring $c: E(G) \rightarrow[r]$ such that, for each $i \in[r]$, the graph $c^{-1}(i)$ of edges coloured $i$ is $F_{i}$-free. It follows from Lemma 6 that there are 'signatures' $S_{2}=g_{2}\left(c^{-1}(2)\right), \ldots, S_{r}=g_{r}\left(c^{-1}(r)\right)$, each with $\left|S_{i}\right|=O\left(n^{2-1 / m_{2}\left(F_{i}\right)}\right) \leqslant O\left(n^{2-1 / m_{2}\left(F_{2}\right)}\right)$ edges, such that $S_{i} \subseteq c^{-1}(i) \subseteq f_{i}\left(S_{i}\right)$ for each $i \in\{2, \ldots, r\}$, where the graph $f_{i}\left(S_{i}\right)$ contains $o\left(n^{v_{F}}\right)$ copies of $F_{i}$. Since each edge of $G$ that lies outside $f_{2}\left(S_{2}\right) \cup \cdots \cup f_{r}\left(S_{r}\right)$ is coloured 1, the intersection of $G$ and the graph

$$
K\left(S_{2}, \ldots, S_{r}\right):=K_{n} \backslash\left(f_{2}\left(S_{2}\right) \cup \cdots \cup f_{r}\left(S_{r}\right)\right)
$$

must be $F_{1}$-free. In particular, the event $G(n, p) \nrightarrow\left(F_{1}, \ldots, F_{r}\right)$ is contained in the union of the events

$$
S_{2} \cup \cdots \cup S_{r} \subseteq G(n, p) \text { and } G(n, p) \cap K\left(S_{2}, \ldots, S_{r}\right) \text { is } F_{1} \text {-free, }
$$


where $\left(S_{2}, \ldots, S_{r}\right)$ ranges over all sequences of 'signatures'. Since the property " $S_{2} \cup \cdots \cup S_{r} \subseteq G$ " is increasing in $G$ and the property " $G \cap K\left(S_{1}, \ldots, S_{r}\right)$ is $F_{1}$-free" is decreasing in $G$, Harris's inequality and the union bound yield

$$
\mathbb{P}\left(G(n, p) \nrightarrow\left(F_{1}, \ldots, F_{r}\right)\right) \leqslant \sum_{\left(S_{2}, \ldots, S_{r}\right)} \mathbb{P}\left(S_{2} \cup \cdots \cup S_{r} \subseteq G(n, p)\right) \cdot \mathbb{P}\left(G(n, p) \cap K\left(S_{2}, \ldots, S_{r}\right) \text { is } F_{1} \text {-free }\right) .
$$

Lemma $\left[\right.$ implies that the graph $K\left(S_{2}, \ldots, S_{r}\right)$ has at least $\delta n^{v_{F_{1}}}$ copies of $F_{1}$, for some constant $\delta>0$ that is independent of $\left(S_{2}, \ldots, S_{r}\right)$, and consequently, following [10], one can derive the bound

$$
\mathbb{P}\left(G(n, p) \cap K\left(S_{2}, \ldots, S_{r}\right) \text { is } F_{1} \text {-free }\right) \leqslant \exp \left(-\delta^{\prime} \cdot \min \left\{n^{v_{I}} p^{e_{I}}: \varnothing \neq I \subseteq F_{1}\right\}\right)
$$

from Janson's inequality.

If one assumes that $p \gg n^{-1 / m_{2}\left(F_{1}, F_{2}\right)} \log n$, then the right-hand side of (3) can be bounded from above by $\exp \left(-\omega\left(n^{2-1 / m_{2}\left(F_{2}\right)} \log n\right)\right)$, whereas there are only $\exp \left(O\left(n^{2-1 / m_{2}\left(F_{2}\right)} \log n\right)\right)$ sequences $\left(S_{2}, \ldots, S_{r}\right)$; thus one may conclude that $G(n, p) \rightarrow\left(F_{1}, \ldots, F_{r}\right)$ with probability very close to 1 , without any further assumptions on $\left(F_{1}, \ldots, F_{r}\right)$. The weaker assumption that $p \gg n^{-1 / m_{2}\left(F_{1}, F_{2}\right)}$ implies only the upper bound $\exp \left(-\omega\left(n^{2-1 / m_{2}\left(F_{2}\right)}\right)\right)$ on the right-hand side of (3) and the challenge is to obtain the estimate

$$
\sum_{\left(S_{2}, \ldots, S_{r}\right)} \mathbb{P}\left(S_{2} \cup \cdots \cup S_{r} \subseteq G(n, p)\right)=\exp \left(O\left(n^{2-1 / m_{2}\left(F_{2}\right)}\right)\right) .
$$

Unfortunately, this estimate is valid only if $p=O\left(n^{-1 / m_{2}\left(F_{2}\right)}\right)$, which we can assume only if $m_{2}\left(F_{1}\right)=$ $m_{2}\left(F_{2}\right)$. This is the essence of why proving the 1-statement of Conjecture 3 is significantly more difficult than proving the 1-statement of Theorem 1.

A first step towards making the above union bound argument more efficient, already taken in [7], is to restrict the family of 'non-Ramsey' colourings that are being considered. To this end, observe that every colouring $c: E(G) \rightarrow[r]$ such that

$$
c^{-1}(i) \text { is } F_{i} \text {-free for every } i \in[r]
$$

may be altered as follows: Every edge of $G$ that is not contained in a copy of $F_{1}$ in $G$ is recoloured 1 . This way we obtain a new colouring $c$ that still satisfies (4) but now each edge of $c^{-1}(2) \cup \cdots \cup c^{-1}(r)$ lies in a copy of $F_{1}$ in $G$. We may thus replace the event " $S_{2} \cup \cdots \cup S_{r} \subseteq G(n, p)$ " in the above argument with the event "each edge of $S_{2} \cup \cdots \cup S_{r}$ lies in a copy of $F_{1}$ in $G(n, p)$ ", which we will abbreviate by $S_{2} \cup \cdots \cup S_{r} \subseteq_{F_{1}} G(n, p)$, and conclude that the event $G(n, p) \nrightarrow\left(F_{1}, \ldots, F_{r}\right)$ is contained in the union of the events

$$
S_{2} \cup \cdots \cup S_{r} \subseteq_{F_{1}} G(n, p) \text { and } G(n, p) \cap K\left(S_{2}, \ldots, S_{r}\right) \text { is } F_{1} \text {-free, }
$$

where again $\left(S_{2}, \ldots, S_{r}\right)$ ranges over all sequences of 'signatures'. Since the property $S_{2} \cup \cdots \cup S_{r} \subseteq_{F_{1}} G$ is still increasing in $G$, in order to complete the argument, it would suffice to prove that

$$
\sum_{\left(S_{2}, \ldots, S_{r}\right)} \mathbb{P}\left(S_{2} \cup \cdots \cup S_{r} \subseteq_{F_{1}} G(n, p)\right)=\exp \left(O\left(n^{2-1 / m_{2}\left(F_{2}\right)}\right)\right) .
$$

Unfortunately, nothing in the spirit of (5D) can be true in general. Indeed, there are pairs of graphs $F_{1}, F_{2}$ such that $m_{2}\left(F_{1}\right)>m_{2}\left(F_{1}, F_{2}\right)>m_{2}\left(F_{2}\right)$, but typically every edge of $G(n, p)$ lies in a copy of $F_{1}$ (for example, this is the case when $F_{1}$ contains an isolated edge), which contradicts (5). However, in the case where $F_{1}$ is strictly balanced w.r.t. $m_{2}\left(\cdot, F_{2}\right)$, one can prove a version 2 of (5) that complements an argument similar to the one we have outlined above; this was achieved in [7].

In order to dispose of the balancedness assumption, we shall restrict our attention only to a subcollection of all copies of $F_{1}$ in $K_{n}$. More precisely, we will require $c^{-1}(1)$ to avoid only a certain family $\mathcal{F}$ of copies of $F_{1}$, which we term typed copies of $F_{1}$. Roughly speaking, every edge of $K_{n}$ will be (randomly) mapped to an edge of $F_{1}$, which we call a type, and a copy $\varphi\left(F_{1}\right)$ of $F_{1}$ in $K_{n}$ will

\footnotetext{
${ }^{2}$ When $m_{2}\left(F_{1}\right)>m_{2}\left(F_{2}\right)$, even if one assumes that $F_{1}$ is strictly balanced w.r.t. $m_{2}\left(., F_{2}\right)$, there is an event of very small probability in $G(n, p)$ that nevertheless blows up the left-hand side of (5) above $\exp \left(O\left(n^{2-1 / m_{2}\left(F_{2}\right)}\right)\right)$. However, after conditioning on the complement of this event, the inequality (5) becomes true.
} 
belong to $\mathcal{F}$ if and only if, for each $e \in E\left(F_{1}\right)$, the type of $\varphi(e)$ is $e$. Moreover, we will replace $G(n, p)$ with a sparser random subgraph of it, whose edge probabilities depend on the types. (We will define both these notions formally in Section 4.) We are going to do both of these things in such a way that the typed copies of $F_{1}$ in this sparser random graph 'behave' like (untyped) copies of a graph that is balanced w.r.t. $m_{2}\left(\cdot, F_{2}\right)$. In particular: (i) the left-hand side of (3) can be still bounded from above by $\exp \left(-\omega\left(n^{2-1 / m_{2}\left(F_{2}\right)}\right)\right)$ and (ii) a version of (5) holds true. Since the left-hand side of (3) is decreasing in both $G(n, p)$ and the family $\mathcal{F}$, whereas each term in the left-hand side of (5) is increasing in both $G(n, p)$ and $\mathcal{F}$, we will have to strike a delicate balance in order to achieve (i) and (ii) simultaneously.

\section{H-TYPED GRAPHS}

For the entirety of this section, suppose that $H$ and $F$ are fixed nonempty graphs.

An important role in our proof is played by weight functions $w: E(H) \rightarrow[1, \infty)$. Given such a function $w$ and a subgraph $I \subseteq H$, we shall use the shorthand $w_{I}:=\sum_{e \in E(I)} w(e)$.

Definition $7((w, F)$-balanced graphs). Given a function $w: E(H) \rightarrow[1, \infty)$, we say that $H$ is $(w, F)$ balanced if, for every edge $e \in E(H)$, we have

$$
\min \left\{v_{I}-w_{I} / m_{2}(H, F): I \subseteq H \text { with } e \in E(I)\right\}=2-1 / m_{2}(F) .
$$

Lemma 8. If $m_{2}(H) \geqslant m_{2}(F)$, then there exists a function $w: E(H) \rightarrow[1, \infty)$ such that $H$ is $(w, F)$ balanced.

Proof. Given a function $w: E(H) \rightarrow[1, \infty)$ and an edge $e \in E(H)$, let us write

$$
r_{e}(w):=\min \left\{v_{I}-w_{I} / m_{2}(H, F): I \subseteq H \text { with } e \in E(I)\right\}-2+1 / m_{2}(F) .
$$

Our goal is then to show that there is a function $w$ such that $r_{e}(w)=0$ for all $e \in E(H)$. To this end, consider the set $\mathcal{W}$ of all functions $w: E(H) \rightarrow[1, \infty)$ that satisfy $r_{e}(w) \geqslant 0$ for all $e \in E(H)$. Note that the constant function $w \equiv 1$ belongs to $\mathcal{W}$, by the definition of $m_{2}(H, F)$, and that $0 \leqslant$ $r_{e}(w) \leqslant 1 / m_{2}(F)-w(e) / m_{2}(H, F)$ for every $e \in E(H)$ and all $w \in \mathcal{W}$. In particular, $\mathcal{W}$ is nonempty and compact and thus the (continuous) map $\mathcal{W} \ni w \mapsto w_{H} \in[0, \infty)$ achieves its maximum at some $\hat{w} \in \mathcal{W}$.

We claim that $r_{e}(\hat{w})=0$ for each $e \in E(H)$. If this were not true and there was an $e \in E(H)$ satisfying $r_{e}(\hat{w})>0$, then, for some sufficiently small $\varepsilon>0$, the function $\tilde{w}$ defined by $\tilde{w}(f)=$ $\hat{w}(f)+\varepsilon \cdot \mathbb{1}[f=e]$ would belong to $\mathcal{W}$, contradicting the maximality of $\hat{w}$.

4.1. $H$-typed graphs. By an $H$-typed graph we mean a graph $\mathcal{G}$ equipped with a type function $\tau_{\mathcal{G}}: E(\mathcal{G}) \rightarrow E(H)$. If $H$ is clear from the context, we shall just say that $\mathcal{G}$ is a typed graph. We shall mostly use calligraphic letters to denote typed graphs; however, every subgraph $I \subseteq H$ will be treated as a typed graph in the natural way, by taking its type function to be the inclusion map from $E(I)$ into $E(H)$. We write $\mathcal{G}^{\prime} \cong \mathcal{G}$ if there is a graph isomorphism between $\mathcal{G}^{\prime}$ and $\mathcal{G}$ that preserves the type of every edge (in this case, we say that $\mathcal{G}$ and $\mathcal{G}^{\prime}$ are typomorphic). We write $\mathcal{G}^{\prime} \preceq \mathcal{G}$ if $\mathcal{G}^{\prime} \subseteq \mathcal{G}$ and $\tau_{\mathcal{G}^{\prime}}(e)=\tau_{\mathcal{G}}(e)$ for all $e \in E\left(\mathcal{G}^{\prime}\right)$. A typed copy of a subgraph $I \subseteq H$ in $\mathcal{G}$ is a typed graph $\mathcal{I} \preceq \mathcal{G}$ such that $\mathcal{I} \cong I$, where $I$ is treated as a typed graph. Finally, for a set $\mathbf{H}$ of (untyped) copies of $H$ in $K_{n}$ and a typed graph $\mathcal{G}$ on the vertex set $[n]$, we write $\mathbf{H}(\mathcal{G})$ for the set of all $\tilde{H} \in \mathbf{H}$ such that $E(\tilde{H}) \subseteq E(\mathcal{G})$ and the typed graph $\tilde{\mathcal{H}}$ obtained by equipping $\tilde{H}$ with the type function $\left.\tau_{\mathcal{G}}\right|_{E(\tilde{H})}$ is typomorphic to $H$.

4.2. Random $H$-typed graphs. We define a random $H$-typed graph $\mathcal{G}(n, p, w)$ as follows.

Definition $9(\mathcal{G}(n, p, w))$. Given $n \in \mathbb{N}, p \in(0,1)$, and $w: E(H) \rightarrow[1, \infty)$, we define $\mathcal{G}(n, p, w)$ to be the random $H$-typed graph $\mathcal{G}$ on the vertex set $[n]$ constructed by the following two-step procedure:

(i) Choose a function $\tau: E\left(K_{n}\right) \rightarrow E(H)$ uniformly at random.

(ii) Include every $e \in E\left(K_{n}\right)$ independently with probability $p^{w(\tau(e))}$ and set $\tau_{\mathcal{G}}=\left.\tau\right|_{E(\mathcal{G})}$. 
We shall be using the following estimate of the upper tail of the number of typed copies of $H$ and its subgraphs in $\mathcal{G}(n, p, w)$; the proof is a straightforward modification of the classical argument of Ruciński and Vince [22].

Lemma 10. Fix a nonempty subgraph $I \subseteq H$ and let $X_{I}$ denote the number of typed copies of $I$ in $\mathcal{G}(n, p, w)$. We have

$$
\mathbb{P}\left(X_{I} \geqslant 2 \mathbb{E}\left[X_{I}\right]\right) \leqslant c \cdot\left(\min _{\varnothing \neq I^{\prime} \subseteq I} n^{v_{I^{\prime}}} p^{w_{I^{\prime}}}\right)^{-1},
$$

for some positive constant $c$ depending only on $H$.

Proof. It is easy to see that

$$
\mathbb{E}\left[X_{I}\right]=\Theta\left(n^{v_{I}} p^{w_{I}}\right)
$$

and

$$
\operatorname{Var}\left[X_{I}\right]=O\left(\max _{\varnothing \neq I^{\prime} \subseteq I} n^{2 v_{I}-v_{I^{\prime}}} p^{2 w_{I}-w_{I^{\prime}}}\right)=O\left(\frac{\mathbb{E}\left[X_{I}\right]^{2}}{\min _{\varnothing \neq I^{\prime} \subseteq I} n^{v_{I^{\prime}}} p^{w_{I^{\prime}}}}\right) .
$$

The assertion now follows from Chebyshev's inequality $\mathbb{P}\left(X_{I} \geqslant 2 \mathbb{E}\left[X_{I}\right]\right) \leqslant \operatorname{Var}\left[X_{I}\right] / \mathbb{E}\left[X_{I}\right]^{2}$.

Lemma 11. Fix a positive $\alpha$ and a family $\mathbf{H}$ of at least $\alpha n^{v_{H}}$ copies of $H$ in $K_{n}$. Then

$$
\mathbb{P}(\mathbf{H}(\mathcal{G}(n, p, w))=\varnothing) \leqslant \exp \left(-c \cdot \min _{\varnothing \neq I \subseteq H} n^{v_{I}} p^{w_{I}}\right)
$$

for some positive constant $c$ depending only on $H$ and $\alpha$.

Proof. For a given copy $C \in \mathbf{H}$, let us write $\mathbb{1}_{C}$ for the indicator variable of the event that $C \in$ $\mathbf{H}(\mathcal{G}(n, p, w))$. Thus $|\mathbf{H}(\mathcal{G}(n, p, w))|=\sum_{C \in \mathbf{H}} \mathbb{1}_{C}$. Observe that $X_{C}$ and $X_{C^{\prime}}$ are independent if $C$ and $C^{\prime}$ are edge-disjoint.

For every $C \in \mathbf{H}$, we have $\mathbb{E}\left[\mathbb{1}_{C}\right] \geqslant p^{w_{H}} / e_{H}^{e_{H}}$, and so

$$
\mu:=\mathbb{E}[|\mathbf{H}(G(n, p, w))|] \geqslant|\mathbf{H}| p^{w_{H}} / e_{H}^{e_{H}} \geqslant \alpha n^{v_{H}} p^{w_{H}} / e_{H}^{e_{H}} .
$$

Define

$$
\Delta:=\sum_{\substack{\left\{C, C^{\prime}\right\} \subseteq \mathbf{H} \\ E\left(C \cap C^{\prime}\right) \neq \varnothing}} \mathbb{E}\left[\mathbb{1}_{C} \mathbb{1}_{C^{\prime}}\right] \quad \text { and } \quad \delta:=\max _{C \in \mathbf{H}} \sum_{\substack{C^{\prime} \in \mathbf{H} \\ E\left(C^{\prime} \cap C\right) \neq \varnothing}} \mathbb{E}\left[\mathbb{1}_{C^{\prime}}\right],
$$

It is easy to check that

$$
\Delta=O\left(\frac{\mu^{2}}{\min _{\varnothing \neq I \subsetneq H} n^{v_{I}} p^{w_{I}}}\right),
$$

where the constants implicit in the $O$-notation may depend on $\alpha$ and on $H$. It is also easy to see that

$$
\delta \leqslant e_{H} n^{v_{H}-2} p^{w_{H}} .
$$

The claim then follows from the following version of Suen's inequality due to Janson [9, Theorem 3]:

$$
\mathbb{P}(|\mathbf{H}(G(n, p, w))|=0) \leqslant \exp \left(-\min \left(\frac{\mu^{2}}{8 \Delta}, \frac{\mu}{6 \delta}, \frac{\mu}{2}\right)\right) .
$$

Note in particular that $\mu / \delta \geqslant \alpha n^{2} / e_{H}^{e_{H}}=\Omega\left(n^{v_{I}} p^{w_{I}}\right)$ for every subgraph $I \subseteq H$ consisting of two vertices and one edge.

\section{Proof of Theorem 4}

Fix nonempty graphs $F_{1}, \ldots, F_{r}$ with $m_{2}\left(F_{1}\right) \geqslant \cdots \geqslant m_{2}\left(F_{r}\right)$ and $m_{2}\left(F_{2}\right) \geqslant 1$. For the sake of brevity, we shall write $H=F_{1}$ and $F=F_{2}$. For each $i \in\{2, \ldots, r\}$, let $C_{i}, f_{i}$, and $g_{i}$ be as given by Lemma 6 applied to the graph $F_{i}$ and to some sufficiently small positive constant $\varepsilon=\varepsilon\left(r, F_{1}, \ldots, F_{r}\right)$; let $C:=\max \left\{C_{2}, \ldots, C_{r}\right\}$.

Given a typed graph $\mathcal{G}$ on $[n]$, we write $\mathcal{G} \nrightarrow\left(H, F_{2}, \ldots, F_{r}\right)$ if there exists a colouring $c: E(\mathcal{G}) \rightarrow[r]$ such that there is neither a typed copy of $H$ in colour 1 , nor an (untyped) copy of $F_{i}$ in colour $i$, for any $i \in\{2, \ldots, r\}$. Note that if $\mathcal{G} \nrightarrow\left(H, F_{2}, \ldots, F_{r}\right)$, then there also exists such a colouring $c$ where 
additionally every edge of $\mathcal{G}$ that is not contained in a typed copy of $H$ has colour 1 . Indeed, we can always recolour such edges in colour 1 without creating any copies of $H$ in $c^{-1}(1)$.

Now, assume that $\mathcal{G} \nrightarrow\left(H, F_{2}, \ldots, F_{r}\right)$ and let $c: E(\mathcal{G}) \rightarrow[r]$ be a colouring that satisfies all of the above conditions. For each $i \in\{2, \ldots, r\}$, let $S_{i}:=g_{i}\left(c^{-1}(i)\right)$ and recall that $S_{i}$ has at most $C n^{2-1 / m_{2}(F)}$ edges. By the definition of $f_{i}$ and $g_{i}$, we have $S_{i} \subseteq c^{-1}(i) \subseteq f_{i}\left(S_{i}\right)$ for every $i \in\{2, \ldots, r\}$. Set $\mathbf{S}:=\left(S_{2}, \ldots, S_{r}\right)$ and let $\mathbf{H}_{\mathbf{S}}$ be the collection of all (untyped) copies of $H$ in the graph $K_{n} \backslash\left(f_{2}\left(S_{2}\right) \cup \cdots \cup f_{r}\left(S_{r}\right)\right)$. Observe crucially that $\mathbf{H}_{\mathbf{S}}(\mathcal{G})=\varnothing$, because every typed copy of $H$ in $\mathbf{H}_{\mathbf{S}}(\mathcal{G})$ would have all of its edges coloured 1, contradicting the choice of the colouring.

By Lemma 8, we can choose a function $w: E(H) \rightarrow[1, \infty)$ such that $H$ is $(w, F)$-balanced. In particular, for every edge $e \in E(H)$, we can fix a subgraph $H_{e} \subseteq H$ containing $e$ such that

$$
v_{H_{e}}-w_{H_{e}} / m_{2}(H, F)=2-1 / m_{2}(F) .
$$

Since every edge in $\mathcal{G}$ of a colour different from 1 is in a typed copy of $H$ in $\mathcal{G}$, we can conclude that for every edge $e \in S_{2} \cup \cdots \cup S_{r}$, there is a typed copy of $H_{f}$ in $\mathcal{G}$ which contains the edge $e$, where $f=\tau_{\mathcal{G}}(e)$. Since $\left|S_{2} \cup \cdots \cup S_{r}\right| \leqslant(r-1) C n^{2-1 / m_{2}(F)}$, the union of these typed copies is a typed subgraph of $\mathcal{G}$ with at most $T(n):=e_{H}(r-1) C n^{2-1 / m_{2}(F)}$ edges.

Let us now summarise the above discussion. For each $i \in\{2, \ldots, r\}$, let $\mathcal{S}_{i}$ comprise the family of all sets of the form $g_{i}(G)$ where $G$ is an $F_{i}$-free graph on $[n]$ and let $\mathcal{S}=\mathcal{S}_{2} \times \cdots \times \mathcal{S}_{r}$. Moreover, let $\mathfrak{W}_{n}$ be the collection of all typed graphs $\mathcal{W}$ with $V(\mathcal{W}) \subseteq[n]$ and $e(\mathcal{W}) \leqslant T(n)$ such that every edge $e \in E(\mathcal{W})$ is contained in a typed copy of $H_{f}$ in $\mathcal{W}$ for some $f \in E(H)$ (where it is not necessarily the case that $\left.f=\tau_{\mathcal{W}}(e)\right)$. What we have shown above can be phrased as follows.

Assertion. If $\mathcal{G}$ is a typed graph on $[n]$ such that $\mathcal{G} \nrightarrow\left(H, F_{2}, \ldots, F_{r}\right)$, then there exists a sequence $\mathbf{S}=\left(S_{2}, \ldots, S_{r}\right) \in \mathcal{S}$, with $S_{2}, \ldots, S_{r}$ pairwise disjoint, and some $\mathcal{W} \in \mathfrak{W}_{n}$ such that:

(1) $S_{2} \cup \cdots \cup S_{r} \subseteq \mathcal{W}$ and $\mathcal{W} \preceq \mathcal{G}$ and

(2) $\mathbf{H}_{\mathbf{S}}(\mathcal{G})=\varnothing$.

We call such a pair $(\mathbf{S}, \mathcal{W})$ a witness for the fact that $\mathcal{G} \nrightarrow\left(H, F_{2}, \ldots, F_{r}\right)$.

Now, suppose that $p \geqslant K n^{-1 / m_{2}(H, F)}$ for a sufficiently large constant $K$. Our goal is to prove that

$$
\mathbb{P}\left(G(n, p) \nrightarrow\left(H, F_{2}, \ldots, F_{r}\right)\right)=o(1) .
$$

As the property $G \nrightarrow\left(H, F_{2}, \ldots, F_{r}\right)$ is monotone decreasing in $G$, we may assume without loss of generality that $p=K n^{-1 / m_{2}(H, F)}$. Since there is a natural coupling of $G(n, p)$ and $\mathcal{G}(n, p, w)$ such that $E(\mathcal{G}(n, p, w)) \subseteq E(G(n, p))$, we may conclude that

$$
\mathbb{P}\left(G(n, p) \nrightarrow\left(H, F_{2}, \ldots, F_{r}\right)\right) \leqslant \mathbb{P}\left(\mathcal{G}(n, p, w) \nrightarrow\left(H, F_{2}, \ldots, F_{r}\right)\right) .
$$

In particular, it suffices to show that the probability that $\mathcal{G}(n, p, w)$ admits a witness $(\mathbf{S}, \mathcal{W})$ for the fact that $\mathcal{G}(n, p, w) \nrightarrow\left(H, F_{2}, \ldots, F_{r}\right)$ is small. This is what we are going to do in the remainder of the proof.

Let $\mathcal{G} \sim \mathcal{G}(n, p, w)$. For a subgraph $I \subseteq H$, we write $X_{I}$ for the number of typed copies of $I$ contained in $\mathcal{G}$. We shall now split the proof into two cases, depending on whether or not $X_{I}$ exceeds $2 \mathbb{E}\left[X_{I}\right]$ for some nonempty $I \subseteq H$.

Case 1. There is a nonempty subgraph $I \subseteq H$ such that $\mathcal{G}$ has more than $2 \mathbb{E}\left[X_{I}\right]$ typed copies of $I$.

The probability that $\mathcal{G}$ is in this case tends to zero by Lemma 10, because $n^{v_{I}} p^{w_{I}} \geqslant K n^{2-1 / m_{2}(F)} \rightarrow$ $\infty$ for every nonempty $I \subseteq H$, by the definition of $(w, F)$-balancedness.

Case 2. For every nonempty subgraph $I \subseteq H, \mathcal{G}$ contains at most $2 \mathbb{E}\left[X_{I}\right]$ typed copies of $I$.

Let us write $\mathfrak{U}_{n} \subseteq \mathfrak{W}_{n}$ for the subset comprising all $\mathcal{W} \in \mathfrak{W}_{n}$ that contain at most $2 \mathbb{E}\left[X_{I}\right]$ typed copies of every nonempty $I \subseteq H$. Note that if $(\mathbf{S}, \mathcal{W})$ is a witness for $\mathcal{G} \nrightarrow\left(H, F_{2}, \ldots, F_{r}\right)$, then necessarily $\mathcal{W} \in \mathfrak{U}_{n}$, since otherwise $\mathcal{G}$ would fall into the first case. Let $Z$ denote the number of witnesses $(\mathbf{S}, \mathcal{W})$ with $\mathcal{W} \in \mathfrak{U}_{n}$. We shall show that $\mathbb{E}[Z]=o(1)$, which, by Markov's inequality, will imply that the probability that $\mathcal{G} \nrightarrow\left(H, F_{2}, \ldots, F_{r}\right)$ tends to zero. 
To this end, we have

$$
\mathbb{E}[Z] \leqslant \sum_{\mathcal{W} \in \mathfrak{U}_{n}} \sum_{\mathbf{S} \in \mathcal{S}(\mathcal{W})} \mathbb{P}\left((\mathbf{S}, \mathcal{W}) \text { is a witness for } \mathcal{G} \nrightarrow\left(H, F_{2}, \ldots, F_{r}\right)\right),
$$

where we write $\mathcal{S}(\mathcal{W})$ for the set of all sequences $\mathbf{S}=\left(S_{2}, \ldots, S_{r}\right) \in \mathcal{S}$ such that $S_{2}, \ldots, S_{r}$ are pairwise edge-disjoint and $S_{2} \cup \cdots \cup S_{r} \subseteq \mathcal{W}$ (in particular, note that $|\mathcal{S}(\mathcal{W})| \leqslant r^{e(\mathcal{W})}$ ). If a pair $(\mathbf{S}, \mathcal{W})$ is a witness for $\mathcal{G} \nrightarrow\left(H, F_{2}, \ldots, F_{r}\right)$, then $\mathcal{W} \preceq \mathcal{G}$ and $\mathbf{H}_{\mathbf{S}}(\mathcal{G})=\varnothing$. Thus

$$
\mathbb{E}[Z] \leqslant \sum_{\mathcal{W} \in \mathfrak{U}_{n}} \sum_{\mathbf{S} \in \mathcal{S}(\mathcal{W})} \mathbb{P}\left(\mathcal{W} \preceq \mathcal{G} \text { and } \mathbf{H}_{\mathbf{S}}(\mathcal{G})=\varnothing\right) .
$$

Given a $\mathcal{W} \in \mathfrak{U}_{n}$ and an $\mathbf{S} \in \mathcal{S}(\mathcal{W})$, let $\mathbf{H}_{\mathbf{S}}^{\mathcal{W}}$ denote the collection of all (untyped) copies of $H$ in $\mathbf{H}_{\mathbf{S}}$ that are edge-disjoint from $\mathcal{W}$. Since $\mathbf{H}_{\mathbf{S}}^{\mathcal{W}}(\mathcal{G}) \subseteq \mathbf{H}_{\mathbf{S}}(\mathcal{G})$ and, crucially, the events $\mathbf{H}_{\mathbf{S}}^{\mathcal{W}}(\mathcal{G})=\varnothing$ and $\mathcal{W} \preceq \mathcal{G}$ are independent, we obtain

$$
\mathbb{E}[Z] \leqslant \sum_{\mathcal{W} \in \mathfrak{U}_{n}} \sum_{\mathbf{S} \in \mathcal{S}(\mathcal{W})} \mathbb{P}\left(\mathcal{W} \preceq \mathcal{G} \text { and } \mathbf{H}_{\mathbf{S}}^{\mathcal{W}}(\mathcal{G})=\varnothing\right)=\sum_{\mathcal{W} \in \mathfrak{U}_{n}} \mathbb{P}(\mathcal{W} \preceq \mathcal{G}) \sum_{\mathbf{S} \in \mathcal{S}(\mathcal{W})} \mathbb{P}\left(\mathbf{H}_{\mathbf{S}}^{\mathcal{W}}(\mathcal{G})=\varnothing\right) .
$$

Since each $f_{i}\left(S_{i}\right)$ contains at most $\varepsilon n^{v_{F_{i}}}$ copies of $F_{i}$, it follows from Ramsey's theorem (Lemma 5) that if $\varepsilon=\varepsilon\left(r, H, F_{2}, \ldots, F_{r}\right)$ is sufficiently small, then $\left|\mathbf{H}_{\mathbf{S}}\right| \geqslant 2 \varepsilon n^{v_{H}}$. Consequently,

$$
\left|\mathbf{H}_{\mathbf{S}}^{\mathcal{W}}\right| \geqslant\left|\mathbf{H}_{\mathbf{S}}\right|-e(\mathcal{W}) \cdot e_{H} \cdot n^{v_{H}-2} \geqslant 2 \varepsilon n^{v_{H}}-O\left(n^{v_{H}-1 / m_{2}(F)}\right) \geqslant \varepsilon n^{v_{H}} .
$$

It thus follows from Lemma 11 and the fact that $H$ is $(w, F)$-balanced that

$$
\mathbb{P}\left(\mathbf{H}_{\mathbf{S}}^{\mathcal{W}}(\mathcal{G})=\varnothing\right) \leqslant \exp \left(-c \cdot \min _{\varnothing \neq I \subseteq H} n^{v_{I}} p^{w_{I}}\right) \leqslant e^{-c K n^{2-1 / m_{2}(F)}}
$$

for some positive constant $c$ that depends only on $H$ and $\varepsilon$. Therefore,

$$
\begin{aligned}
\mathbb{E}[Z] & \leqslant \sum_{\mathcal{W} \in \mathfrak{U}_{n}} \mathbb{P}(\mathcal{W} \preceq \mathcal{G}) \sum_{\mathbf{S} \in \mathcal{S}(\mathcal{W})} e^{-c K n^{2-1 / m_{2}(F)}} \\
& \leqslant \sum_{\mathcal{W} \in \mathfrak{U}_{n}} \mathbb{P}(\mathcal{W} \preceq \mathcal{G}) \cdot r^{e(\mathcal{W})} \cdot e^{-c K n^{2-1 / m_{2}(F)}} \\
& \leqslant e^{-c K n^{2-1 / m_{2}(F)} / 2} \cdot \sum_{\mathcal{W} \in \mathfrak{U}_{n}} \mathbb{P}(\mathcal{W} \preceq \mathcal{G}),
\end{aligned}
$$

where we have used that every $\mathcal{W} \in \mathfrak{U}_{n}$ has at most $T(n)$ edges and thus, for sufficiently large $K$,

$$
e(\mathcal{W}) \log r \leqslant T(n) \log r \leqslant e_{H}(r-1) C n^{2-1 / m_{2}(F)} \log r \leqslant c K n^{2-1 / m_{2}(F)} / 2 .
$$

It remains to estimate the sum in the right-hand side of (8). To this end, for a given $k \in \mathbb{N}$, let $\mathfrak{U}_{n, k}$ be the set of all $\mathcal{W} \in \mathfrak{U}_{n}$ that can be written as a union $\mathcal{H}_{1} \cup \cdots \cup \mathcal{H}_{k}$ of $k$ typed graphs with $V\left(\mathcal{H}_{i}\right) \subseteq[n]$, each of which is typomorphic to $H_{f}$ for some $f \in E(H)$, but not as a union of fewer than $k$ such graphs. Letting $U_{k}$ count the number of $\mathcal{W} \in \mathfrak{U}_{n, k}$ such that $\mathcal{W} \preceq \mathcal{G}$, we now have

$$
\sum_{\mathcal{W} \in \mathfrak{U}_{n}} \mathbb{P}(\mathcal{W} \preceq \mathcal{G})=\sum_{k \leqslant T(n)} \sum_{\mathcal{W} \in \mathfrak{U}_{n, k}} \mathbb{P}(\mathcal{W} \preceq \mathcal{G})=\sum_{k \leqslant T(n)} \mathbb{E}\left[U_{k}\right] .
$$

Claim 12. There is a constant $c_{H}$ that depends only on $H$ such that, for every $k$,

$$
\mathbb{E}\left[U_{k}\right] \leqslant\left(\frac{c_{H} \cdot K^{w_{H}} n^{2-1 / m_{2}(F)}}{k}\right)^{k} .
$$

Proof of Claim. Since the only member of $\mathfrak{U}_{n, 0}$ is the empty graph, we have $U_{0}=1$. We now show that, for every $k \geqslant 1$,

$$
\mathbb{E}\left[U_{k}\right] \leqslant \frac{1}{k} \cdot \mathbb{E}\left[U_{k-1}\right] \cdot e_{H} 2^{1+v_{H}+e_{H}} \cdot K^{w_{H}} n^{2-1 / m_{2}(F)} .
$$

It is easy to see that (10) implies that, for every $k$,

$$
\mathbb{E}\left[U_{k}\right] \leqslant \frac{1}{k !}\left(e_{H} 2^{1+v_{H}+e_{H}} \cdot K^{w_{H}} n^{2-1 / m_{2}(F)}\right)^{k},
$$


which in turn implies the assertion of the claim. Thus we only need to prove (10).

To this end, consider some $\mathcal{W} \in \mathfrak{U}_{n, k}$ and let $\mathcal{H}_{1} \cup \cdots \cup \mathcal{H}_{k}$ be some representation of $\mathcal{W}$ as the union of typed graphs, each of which is typomorphic to some $H_{f} 3$ Observe that, for every $i \in[k]$, the typed graph $\mathcal{W}_{i}=\bigcup_{j \in[k] \backslash\{i\}} \mathcal{H}_{j}$ belongs to $\mathfrak{U}_{n, k-1}$. Moreover, all these typed graphs are distinct, because every $\mathcal{H}_{i}$ contains an edge that is not covered by the union of all the other $\mathcal{H}_{j}$ for $j \neq i$. In other words, for every $\mathcal{W} \in \mathfrak{U}_{n, k}$, there are at least 4 distinct typed graphs $\mathcal{W}_{i} \in \mathfrak{U}_{n, k-1}$ such that $\mathcal{W}=\mathcal{W}_{i} \cup \mathcal{H}_{i}$ for some typed copy $\mathcal{H}_{i}$ of some $H_{f}$ with $f \in E(H)$. Denoting, for each $I \subseteq H$, the set of all typed graphs with vertices from $[n]$ that are typomorphic to $I$ by $\mathfrak{C}_{n}(I)$, have

$$
k \cdot \mathbb{E}\left[U_{k}\right]=k \sum_{\mathcal{W} \in \mathfrak{U}_{n, k}} \mathbb{P}(\mathcal{W} \preceq \mathcal{G}) \leqslant \sum_{\mathcal{W}^{\prime} \in \mathfrak{U}_{n, k-1}} \sum_{f \in E(H)} \sum_{\mathcal{H}^{\prime} \in \mathfrak{C}\left(H_{f}\right)} \mathbb{P}\left(\mathcal{W}^{\prime} \cup \mathcal{H}^{\prime} \preceq \mathcal{G}\right),
$$

where we further require that the type function of each $\mathcal{H}^{\prime}$ in the last sum agrees with that of $\mathcal{W}^{\prime}$ on the intersection $\mathcal{W}^{\prime} \cap \mathcal{H}^{\prime}$ (otherwise $\mathcal{W}^{\prime} \cup \mathcal{H}^{\prime}$ would not be a well defined typed graph). Fix arbitrary $\mathcal{W}^{\prime} \in \mathfrak{U}_{n, k-1}$ and $f \in E(H)$ and observe that, for every $\mathcal{H}^{\prime} \in \mathfrak{C}\left(H_{f}\right)$, we have

$$
\mathbb{P}\left(\mathcal{W}^{\prime} \cup \mathcal{H}^{\prime} \preceq \mathcal{G}\right)=\mathbb{P}\left(\mathcal{W}^{\prime} \preceq \mathcal{G}\right) \cdot \mathbb{P}\left(\mathcal{H}^{\prime} \backslash\left(\mathcal{H}^{\prime} \cap \mathcal{W}^{\prime}\right) \preceq \mathcal{G}\right) .
$$

By first specifying the intersection $\mathcal{I}=\mathcal{W}^{\prime} \cap \mathcal{H}^{\prime}$, which is necessarily a typed copy in $\mathcal{W}^{\prime}$ of some $I \subseteq H_{f}$, we have

$$
\sum_{\mathcal{H}^{\prime} \in \mathfrak{C}_{n}\left(H_{f}\right)} \mathbb{P}\left(\mathcal{W}^{\prime} \cup \mathcal{H}^{\prime} \preceq \mathcal{G}\right)=\sum_{I \subseteq H_{f}} \sum_{\substack{\mathcal{I} \in \mathfrak{C}_{n}(I) \\ \mathcal{I} \preceq \mathcal{W}^{\prime}}} \sum_{\substack{\mathcal{H}^{\prime} \in \mathfrak{C}_{n}\left(H_{f}\right) \\ \mathcal{W}^{\prime} \cap \mathcal{H}^{\prime}=\mathcal{I}}} \mathbb{P}\left(\mathcal{W}^{\prime} \preceq \mathcal{G}\right) \cdot \mathbb{P}\left(\mathcal{H}^{\prime} \backslash \mathcal{I} \preceq \mathcal{G}\right),
$$

Because for every $\mathcal{H}^{\prime} \in \mathfrak{C}_{n}\left(H_{f}\right)$, every $I \subseteq H_{f}$, and every $\mathcal{I} \in \mathfrak{C}_{n}(I)$ with $\mathcal{I} \preceq \mathcal{H}^{\prime}$, we have $\mathbb{P}\left(\mathcal{H}^{\prime} \backslash \mathcal{I} \preceq\right.$ $\mathcal{G})=p^{w_{H_{f}}-w_{I}}$, and since by the definition of $\mathfrak{U}_{n}$, there are at most $2 \mathbb{E}\left[X_{I}\right] \leqslant 2 n^{v_{I}} p^{w_{I}}$ typed copies of $I$ in $\mathcal{W}^{\prime}$, we get

$$
\begin{aligned}
\sum_{\mathcal{H}^{\prime} \in \mathfrak{C}_{n}\left(H_{f}\right)} \mathbb{P}\left(\mathcal{W}^{\prime} \cup \mathcal{H}^{\prime} \preceq \mathcal{G}\right) & \leqslant \mathbb{P}\left(\mathcal{W}^{\prime} \preceq \mathcal{G}\right) \cdot \sum_{I \subseteq H_{f}} 2 n^{v_{I}} p^{w_{I}} \cdot n^{v_{H_{f}}-v_{I}} \cdot p^{w_{H_{f}}-w_{I}} \\
& \leqslant \mathbb{P}\left(\mathcal{W}^{\prime} \preceq \mathcal{G}\right) \cdot 2^{1+v_{H_{f}}+e_{H_{f}}} \cdot n^{v_{H_{f}}} p^{w_{H_{f}}} .
\end{aligned}
$$

Recalling that $v_{H_{f}}-w_{H_{f}} / m_{2}(H, F)=2-1 / m_{2}(F)$ by the definition of $H_{f}$, our assumption that $p=K n^{-1 / m_{2}(H, F)}$ gives $n^{v_{H}} p^{w_{H_{f}}}=K^{w_{H_{f}}} n^{2-1 / m_{2}(F)}$. We may thus conclude that

$$
\sum_{\mathcal{H}^{\prime} \in \mathfrak{C}_{n}\left(H_{f}\right)} \mathbb{P}\left(\mathcal{W}^{\prime} \cup \mathcal{H}^{\prime} \preceq \mathcal{G}\right) \leqslant \mathbb{P}\left(\mathcal{W}^{\prime} \preceq \mathcal{G}\right) \cdot 2^{1+v_{H_{f}}+e_{H_{f}}} \cdot K^{w_{H}} n^{2-1 / m_{2}(F)} .
$$

Substituting this bound into (11), we obtain

$$
\begin{aligned}
k \cdot \mathbb{E}\left[U_{k}\right] & \leqslant \sum_{\mathcal{W}^{\prime} \in \mathfrak{U}_{n, k-1}} \sum_{f \in E(H)} \mathbb{P}\left(\mathcal{W}^{\prime} \preceq \mathcal{G}\right) \cdot 2^{1+v_{H_{f}}+e_{H_{f}}} \cdot K^{w_{H_{f}}} n^{2-1 / m_{2}(F)} \\
& =\mathbb{E}\left[U_{k-1}\right] \cdot \sum_{f \in E(H)} 2^{1+v_{H_{f}}+e_{H}} \cdot K^{w_{H}} n^{2-1 / m_{2}(F)} \\
& \leqslant \mathbb{E}\left[U_{k-1}\right] \cdot e_{H} 2^{1+v_{H}+e_{H}} \cdot K^{w_{H}} n^{2-1 / m_{2}(F)} .
\end{aligned}
$$

Dividing through by $k$, we obtain (10).

Combining (9) and the claim, we obtain

$$
\sum_{\mathcal{W} \in \mathfrak{U}_{n}} \mathbb{P}(\mathcal{W} \preceq \mathcal{G}) \leqslant \sum_{k \leqslant T(n)}\left(\frac{c_{H} \cdot K^{w_{H}} n^{2-1 / m_{2}(F)}}{k}\right)^{k} \leqslant(T(n)+1) \cdot\left(\frac{c_{H} \cdot K^{w_{H}} n^{2-1 / m_{2}(F)}}{T(n)}\right)^{T(n)}
$$

\footnotetext{
${ }^{3}$ Note that this representation is not necessarily unique.

4 "At least" since, again, $\mathcal{W}$ can have more than one representation as $\mathcal{H}_{1} \cup \cdots \cup \mathcal{H}_{k}$.
} 
where the last inequality follows from the fact that the function $x \mapsto(c / x)^{x}$ is increasing for $0<x \leqslant c / e$ and that $T(n)=e_{H}(r-1) C n^{2-1 / m_{2}(F)} \leqslant c_{H} \cdot K^{w_{H}} n^{2-1 / m_{2}(F)} / e$ if $K$ is large. This yields

$$
\sum_{\mathcal{W} \in \mathfrak{U}_{n}} \mathbb{P}(\mathcal{W} \preceq \mathcal{G}) \leqslant\left(\frac{2 c_{H} K^{w_{H}}}{e_{H}(r-1) C}\right)^{e_{H}(r-1) C n^{2-1 / m_{2}(F)}} \leqslant e^{O(\log K) n^{2-1 / m_{2}(F)}},
$$

which, together with (8), implies that $\mathbb{E}[Z] \rightarrow 0$, provided that $K$ is sufficiently large. This completes the proof.

\section{Concluding Remarks}

While, even before our work, the 1-statement of Conjecture 3 was known to be true up to a $\log n$ factor, the situation with the 0 -statement is quite different. So far it has only been verified in the case where all the graphs $F_{1}, \ldots, F_{r}$ are either cycles [12] or complete graphs [16]. The general case, however, seems to be rather difficult. A criterion which reduces the 0 -statement of Conjecture 3 to a purely deterministic question, a potentially fruitful approach, was given in [7]. We now present this reduction.

Given graphs $F_{1}$ and $F_{2}$, let $\mathbf{F}\left(F_{1}, F_{2}\right)$ be the family of all graphs $F$ with the following property: There exists a copy $F_{2}^{\prime}$ of $F_{2}$ in $F$ and an edge $e_{0} \in E\left(F_{2}^{\prime}\right)$ such that, for each $e \in E\left(F_{2}^{\prime}\right) \backslash\left\{e_{0}\right\}$, there is a copy $F_{1}^{e}$ of $F_{1}$ in $F$ containing $e$ and

$$
E(F)=E\left(F_{2}^{\prime}\right) \cup \bigcup_{e \in E\left(F_{2}^{\prime}\right) \backslash\left\{e_{0}\right\}} E\left(F_{1}^{e}\right) ;
$$

we shall call such an $e_{0}$ an attachment edge. Note that the graphs $F_{1}^{e}$ need not be disjoint (in fact they are even not required to be distinct). Intuitively, every graph in $\mathbf{F}\left(F_{1}, F_{2}\right)$ is formed from a copy of $F_{2}$ by gluing copies of $F_{1}$ on all of its edges except some edge $e_{0}$. Let us call a graph $F \in \mathbf{F}\left(F_{1}, F_{2}\right)$ generic if every $F_{1}^{e}$ intersects $F_{2}^{\prime}$ only in the edge $e$ (and no vertices other than the endpoints of $e$ ) and its remaining vertices are disjoint from all the other $F_{1}^{e^{\prime}}$ with $e^{\prime} \neq e$. Note that there can be up to $e\left(F_{2}\right) \cdot e\left(F_{1}\right)^{e\left(F_{2}\right)-1}$ different generic graphs.

The main property we require from the family $\mathbf{F}\left(F_{1}, F_{2}\right)$ is that these generic graphs are the 'sparsest' among all graphs in $\mathbf{F}\left(F_{1}, F_{2}\right)$. In particular, we say that $\mathbf{F}\left(F_{1}, F_{2}\right)$ is asymmetric-balanced if the following two conditions are met for every $F \in \mathbf{F}\left(F_{1}, F_{2}\right)$ and every $H \subseteq F$ with $V(H) \subsetneq V(F)$ containing an attachment edge:

(1) We have

(2) Moreover, if

$$
\frac{e(F)-e(H)}{v(F)-v(H)} \geqslant m_{2}\left(F_{1}, F_{2}\right)
$$

$$
\frac{e(F)-e(H)}{v(F)-v(H)}=m_{2}\left(F_{1}, F_{2}\right),
$$

then $F$ is generic and $H$ contains a single edge (the attachment edge).

Theorem $13([7])$. Let $F_{1}$ and $F_{2}$ be graphs such that $m_{2}\left(F_{1}\right) \geqslant m_{2}\left(F_{2}\right)>1$ and suppose that the following holds:

(i) $F_{1}$ and $F_{2}$ are strictly 2-balanced,

(ii) $F_{1}$ is strictly balanced w.r.t. $m_{2}\left(\cdot, F_{2}\right)$,

(iii) $\mathbf{F}\left(F_{1}, F_{2}\right)$ is asymmetric-balanced, and

(iv) for every graph $G$ such that

$$
\max _{G^{\prime} \subseteq G} \frac{e\left(G^{\prime}\right)}{v\left(G^{\prime}\right)} \leqslant m_{2}\left(F_{1}, F_{2}\right)
$$

we have $G \nrightarrow\left(F_{1}, F_{2}\right)$.

Then there exists $c>0$ such that if $p \leqslant c n^{-1 / m_{2}\left(F_{1}, F_{2}\right)}$, then

$$
\lim _{n \rightarrow \infty} \mathbb{P}\left(G(n, p) \rightarrow\left(F_{1}, F_{2}\right)\right)=0 .
$$


A minor modification of the proof of Theorem 13 shows that one can further drop the requirement in (i) that $F_{1}$ is strictly 2-balanced. Therefore, in order to prove the 0 -statement of Conjecture 3, it is enough to consider a strictly 2-balanced subgraph $F_{2}^{\prime} \subseteq F_{2}$ with $m_{2}\left(F_{2}^{\prime}\right)=m_{2}\left(F_{2}\right)$ and a subgraph $F_{1}^{\prime} \subseteq F_{1}$ that is strictly balanced w.r.t $m_{2}\left(\cdot, F_{2}^{\prime}\right)$ and satisfies $m_{2}\left(F_{1}^{\prime}, F_{2}^{\prime}\right)=m_{2}\left(F_{1}, F_{2}^{\prime}\right)$, and show that conditions (iii) and (iv) in Theorem 13 hold. As an exercise, we invite the reader to show this in the case where $m_{2}\left(F_{1}\right)=m_{2}\left(F_{2}\right)$ (for part (iv) see, e.g., the appendix of [18]). In this case, it turns out that if $F_{1}^{\prime} \subseteq F_{1}$ is chosen in the manner described above, then it is also strictly 2-balanced and $m_{2}\left(F_{1}^{\prime}\right)=m_{2}\left(F_{2}^{\prime}\right)$; in particular, one can use Theorem 13 without any modifications. Unfortunately, the general case remains wide open.

Finally, let us mention that the proof of Theorem 4 transfers to the setting of uniform hypergraphs with almost no changes. However, unlike for graphs, in the case of hypergraphs of uniformity larger than two, even in the symmetric case (i.e., $F_{1}=\cdots=F_{r}$ ) a complete characterisation of the threshold functions is not known. We refer the interested reader to [7, 17] for further details.

\section{REFERENCES}

[1] J. Balogh, R. Morris, and W. Samotij. Independent sets in hypergraphs. J. Amer. Math. Soc., 28(3):669-709, 2015.

[2] J. Balogh, R. Morris, and W. Samotij. The method of hypergraph containers. In Proceedings of the International Congress of Mathematicians-Rio de Janeiro 2018. Vol. IV. Invited lectures, pages 3059-3092. World Sci. Publ., Hackensack, NJ, 2018.

[3] D. Conlon, W. T. Gowers, W. Samotij, and M. Schacht. On the KŁR conjecture in random graphs. Israel J. Math., 203(1):535-580, 2014.

[4] P. Frankl and V. Rödl. Large triangle-free subgraphs in graphs without $K_{4}$. Graphs Combin., 2(2):135-144, 1986.

[5] E. Friedgut and M. Krivelevich. Sharp thresholds for certain Ramsey properties of random graphs. Random Structures Algorithms, 17(1):1-19, 2000.

[6] E. Friedgut, V. Rödl, A. Ruciński, and P. Tetali. A sharp threshold for random graphs with a monochromatic triangle in every edge coloring. Mem. Amer. Math. Soc., 179(845):vi+66, 2006.

[7] L. Gugelmann, R. Nenadov, Y. Person, N. Škorić, A. Steger, and H. Thomas. Symmetric and asymmetric Ramsey properties in random hypergraphs. Forum Math. Sigma, 5:e28, 47, 2017.

[8] R. Hancock, K. Staden, and A. Treglown. Independent sets in hypergraphs and Ramsey properties of graphs and the integers. SIAM J. Discrete Math., 33(1):153-188, 2019.

[9] S. Janson. New versions of Suen's correlation inequality. In Proceedings of the Eighth International Conference "Random Structures and Algorithms" (Poznan, 1997), volume 13, pages 467-483, 1998.

[10] S. Janson, T. Łuczak, and A. Ruciński. An exponential bound for the probability of nonexistence of a specified subgraph in a random graph. In Random graphs '87 (Poznań, 1987), pages 73-87. Wiley, Chichester, 1990.

[11] S. Janson, T. Łuczak, and A. Ruciński. Random graphs. Wiley-Interscience Series in Discrete Mathematics and Optimization. Wiley-Interscience, New York, 2000.

[12] Y. Kohayakawa and B. Kreuter. Threshold functions for asymmetric Ramsey properties involving cycles. Random Structures Algorithms, 11(3):245-276, 1997.

[13] Y. Kohayakawa, T. Łuczak, and V. Rödl. On $K^{4}$-free subgraphs of random graphs. Combinatorica, 17(2):173-213, 1997.

[14] Y. Kohayakawa, M. Schacht, and R. Spöhel. Upper bounds on probability thresholds for asymmetric Ramsey properties. Random Structures Algorithms, 44(1):1-28, 2014.

[15] T. Łuczak, A. Ruciński, and B. Voigt. Ramsey properties of random graphs. J. Combin. Theory Ser. B, 56(1):55-68, 1992.

[16] M. Marciniszyn, J. Skokan, R. Spöhel, and A. Steger. Asymmetric Ramsey properties of random graphs involving cliques. Random Structures Algorithms, 34(4):419-453, 2009.

[17] R. Nenadov, Y. Person, N. Škorić, and A. Steger. An algorithmic framework for obtaining lower bounds for random Ramsey problems. J. Combin. Theory Ser. B, 124:1-38, 2017.

[18] R. Nenadov and A. Steger. A short proof of the random Ramsey theorem. Combin. Probab. Comput., 25(1):130-144, 2016.

[19] V. Rödl and A. Ruciński. Lower bounds on probability thresholds for Ramsey properties. In Combinatorics, Paul Erdôs is eighty, Vol. 1, Bolyai Soc. Math. Stud., pages 317-346. János Bolyai Math. Soc., Budapest, 1993.

[20] V. Rödl and A. Ruciński. Random graphs with monochromatic triangles in every edge coloring. Random Structures Algorithms, 5(2):253-270, 1994.

[21] V. Rödl and A. Ruciński. Threshold functions for Ramsey properties. J. Amer. Math. Soc., 8(4):917-942, 1995. 
[22] A. Ruciński and A. Vince. Balanced graphs and the problem of subgraphs of random graphs. In Proceedings of the sixteenth Southeastern international conference on combinatorics, graph theory and computing (Boca Raton, Fla., 1985), volume 49, pages 181-190, 1985.

[23] D. Saxton and A. Thomason. Hypergraph containers. Invent. Math., 201(3):925-992, 2015.

[24] M. Schacht and F. Schulenburg. Sharp thresholds for Ramsey properties of strictly balanced nearly bipartite graphs. Random Structures Algorithms, 52(1):3-40, 2018.

Frank Mousset, School of Mathematical Sciences, Tel Aviv University, Tel Aviv 6997801, Israel

E-mail address: moussetfrank@gmail.com

Rajko Nenadov, Department of Computer Science, ETH Zurich, 8092 Zürich, Switzerland

E-mail address: rnenadov@inf.ethz.ch

Wojciech Samotiu, School of Mathematical Sciences, Tel Aviv University, Tel Aviv 6997801, Israel

E-mail address: samotij@post.tau.ac.il 